\title{
Octagonal Shaped Metamaterial Absorber Based Energy Harvester
}

\author{
Fatih Ozkan ALKURT ${ }^{1}$, Olcay ALTINTAS ${ }^{1}$, Mehmet BAKIR ${ }^{2}$, Ahmet TAMER ${ }^{1}$, \\ Faruk KARADAG ${ }^{3}$, Mehmet BAGMANCI ${ }^{1}$, Muharrem KARAASLAN ${ }^{1}$, Emin $^{\text {UNAL }}{ }^{1}$, \\ Oguzhan AKGOL ${ }^{1}$
}

\author{
${ }^{1}$ Department of Electrical and Electronics Engineering, Iskenderun Technical University,31200, Iskenderun, Hatay, Turkey \\ ${ }^{2}$ Department of Computer Engineering, Faculty of Engineering and Architecture, Bozok University, Yozgat, Turkey \\ ${ }^{3}$ Department of Physics, Cukurva University, Adana, Turkey
}

crossref http://dx.doi.org/10.5755/j01.ms.24.3.18625

\section{Received 20 July 2017; accepted 10 November 2017}

\begin{abstract}
In this study, a novel octagonal shaped metamaterial based broadband absorber is proposed for energy harvesting at WIMAX wireless communication band. The proposal has an absorption of $99.97 \%$ at $5.5 \mathrm{GHz}$ with a bandwidth of $1 \mathrm{GHz}$ between $5 \mathrm{GHz}$ and $6 \mathrm{GHz}$ in Transverse Electric (TE) mode. Transverse Electric (TE), Transverse Magnetic (TM) and Transverse Electromagnetic (TEM) modes are studied numerically in this paper. In TM and TEM modes, proposed structure has absorption of nearly $96 \%$ and $98 \%$ respectively at $6 \mathrm{GHz}$. The proposed structure is polarization angle independent which is important for energy harvesting applications. Energy harvesting application is realized by using resistive loads on the design, simulations are parametrically studied on resistive loads and experimental study is realized for reflection coefficient measurement and compared with simulated results. Both experimental and simulated results are complying with each other. The proposed structure has different usage areas in stealth areas, detection and imaging. Keywords: metamaterial absorber, energy harvesting, wireless communication.
\end{abstract}

\section{INTRODUCTION}

Metamaterials (MMs) are artificially engineered materials which show specific electromagnetic (EM) properties that cannot be found inherently in the nature. After the proposal of the Victor Veselago in 1967 [1], in 2000, first metamaterial was developed artificially with negative permeability and negative permittivity [2] by using periodic metal resonators. MMs have potential applications in cloaking [3, 4], super lensing [5], antennas [6 - 8], sensors [9-12], metamaterial based harvesters and absorbers [13-16]. In brief, varied kinds of metamaterials with many applications were analyzed for various applications [17-20]. First metamaterial based absorber was introduced by Landy in [15] which is an investigation of unity absorption by using two resonators to captive radiated electromagnetic field. Perfect absorption can be accomplished by arranging the periodic structures in a way that by modification of complex permittivity and permeability for fully absorb electromagnetic energy [21]. Actually, there are several important works in the literature [22-25] that is related with perfect absorption. For example, Bilotti et al. [22] proposed split ring array resonator to absorb microwave energy. $\mathrm{Zu}$ et al. [23] suggested that polarization insensitive metamaterial absorber and this study includes fabrication. In another study [24], polarization and incident angle insensitive dualband metamaterial absorber is investigated which is based on isotropic resonators. Cheng and Yang proposed a metamaterial absorber composed of two resonators and a metal wire for increasing the absorption rate in the microwave region in [25]. Yunsong, et al. [26] proposed a subwavelength resolution microwave camera based on a metamaterial absorber and they investigated microwave imaging techniques.

In this paper, a novel octagonal shape metamaterial based absorber and energy harvester is designed for Worldwide Interoperability for Microwave Access (WIMAX), wireless communication band with wide band absorption nearly $1 \mathrm{GHz}$ in the resonance region have been proposed. Absorption has been verified by simulation and experimentally and results are compliant with each other. Simulation part of study have been realised by Finite Integration Technique (FIT) based Simulator that supports under Transverse Electric (TE), Transverse Magnetic (TM), and Transverse Electromagnetic (TEM) modes. The proposed structure provides a wide bandwidth and has polarization angle independency.

\section{DESIGN AND NUMERICAL SETUP}

Design of the proposed structure have been realised by CST Microwave Studio and experimental study results by using parametric and genetic algorithm tools. For the TE and TM mode simulations, boundaries are chosen as unit cell in both X, Y directions and open (add space) in the $\mathrm{Z}$ direction. For TEM mode simulation, boundary condition of simulation program has been set to Electric, Magnetic and open add space in simulation. Design details of the proposed structure is given in Fig. 1 a and Table 1. In Fig. 1 b., unit cell of view of the proposed structure is seen. Back side of the proposed design is covered by couple layer to prevent transmission and reduce reflection coefficient level. Proposed structure is placed on FR4 substrate which is

\footnotetext{
*Corresponding author. Tel.: +90-5314563210; fax: +90-326-6135613.
}

E-mailaddress: muharrem.karaaslan@iste.edu.tr (M. Karaaslan) 
$24 \mathrm{~mm} \times 24 \mathrm{~mm}$ in $\mathrm{X}$ and $\mathrm{Y}$ dimensions and it has a thickness of $3.2 \mathrm{~mm}$ to increase absorption and bandwidth. Resonator is made up of copper that has a conductivity of $5.8 \cdot 10^{7} \mathrm{~S} / \mathrm{m}$ and it has a thickness of $0.035 \mathrm{~mm}$.

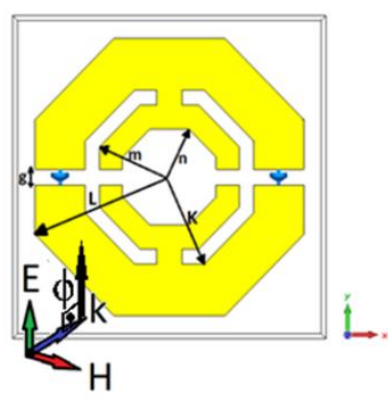

a

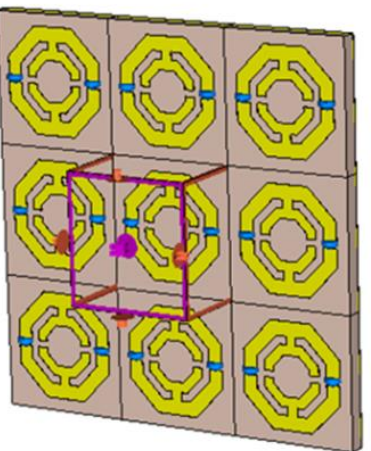

b
Fig. 1. A novel octagonal metamaterial based absorber design with resistive loads with incident angle phi $(\phi) 90$ deg: a-unit cell structure; $b$ - metamaterial full structure

Table 1. Parameters of the proposed structure

\begin{tabular}{|c|c|c|c|c|c|}
\hline Load & $\mathrm{K}, \mathrm{mm}$ & $\mathrm{L}, \mathrm{mm}$ & $\mathrm{m}, \mathrm{mm}$ & $\mathrm{n}, \mathrm{mm}$ & $\mathrm{g}, \mathrm{mm}$ \\
\hline $325 \mathrm{ohm}$ & 105 & 7.6 & 6 & 4 & 1.2 \\
\hline
\end{tabular}

The absorption $A(w)$ term can be defined according to equation $A(w)=1-R(w)-T(w)$, where $R(\mathrm{w})$ and $T(\mathrm{w})$ indicates reflection and transmission coefficients of the proposed structure. The absorption formula can be minimized by using a metal back plate to prevent transmission, so $T(w)=\left|S_{21}\right|^{2}=0$. As a result of using metal back plate, absorption formula revised to $A(w)=1-$ $R(w)=1-\left|S_{11}\right|^{2}$. From the revised formula, when the reflection is minimum so the absorption will be maximum and transmission coefficient is assumed to be zero in this study. In order to explain design properties of the proposed structure, first of all effect of the resistive load which is used as a lumped element on absorption have been investigated. Absorption versus frequency between $3 \mathrm{GHZ}$ and $7 \mathrm{GHz}$ when resistor is not used on the structure has been given in Fig. 2. In this usage, simulation results show that the structure has low absorptivity nearly $33 \%$ between $5 \mathrm{GHz}$ and $6 \mathrm{GHz}$ band in the TE mode.

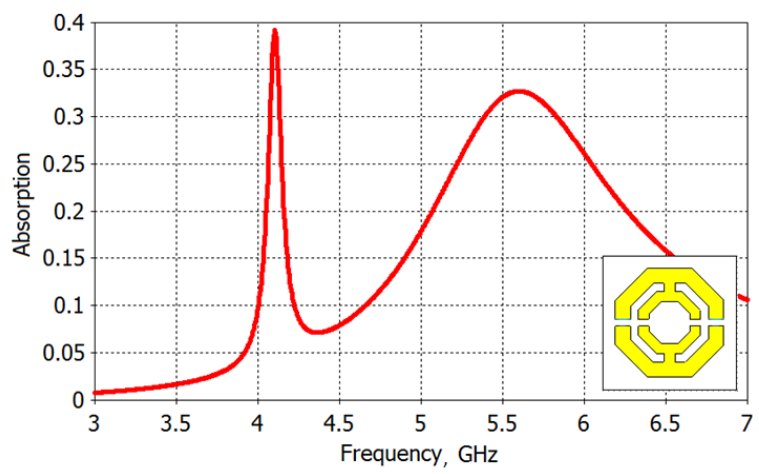

Fig. 2. Absorption of the octagonal metamaterial absorber without resistive loads

In order to find proper resistive load values a parametric study have been realised by simulation program as shown in
Fig. 3. According to simulation study results, best bandwidth has been obtained in $325 \mathrm{ohm}$ lumped element resistance value. As shown in Fig. 3, lumped element resistive load values have been analysed between $200 \mathrm{ohm}$ and $1000 \mathrm{ohm}$ values at 10 different values. Since perfect absorption together with desired bands have been supplied when 325 ohm resistive load, this value becomes the design input of the proposed structure.

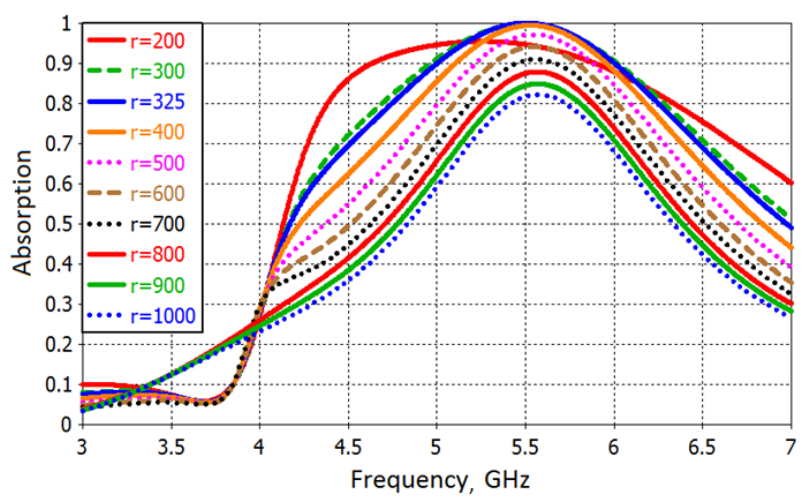

Fig. 3. Parametric study for the resistive load between $200 \mathrm{ohm}$ and $1000 \mathrm{ohm}$ at 6 different points between $3 \mathrm{GHz}$ and $7 \mathrm{GHz}$

Another simulation study has been realized to give more information about design parameters of the proposed structure that absorption value according to $\mathrm{K}$ and $\mathrm{L}$ values of the proposed structure is studied. Obtained simulation results are presented in Fig. 4. and Fig. 5. First of all, L value and resistive load values have been fixed to $10.5 \mathrm{~mm}$ and $325 \mathrm{ohm}$ to see effects of $\mathrm{L}$ parameter in absorption. $\mathrm{L}$ parameter is now adjusted between $6.8 \mathrm{~mm}$ and $8.4 \mathrm{~mm}$ in 5 different values as will be presented in Fig. 4 .

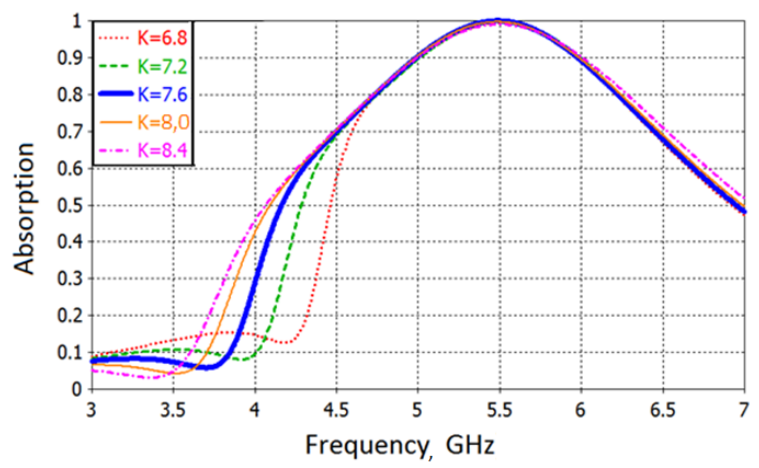

Fig. 4. Parametric result for the $\mathrm{K}$ value, $\mathrm{L}=10.5 \mathrm{~mm}$, $\mathrm{R}=325 \mathrm{ohm}$

According to simulation study results, $\mathrm{K}$ value is decided to be $7.6 \mathrm{~mm}$ due to perfect absorption and desired frequency bands. After simulating $\mathrm{K}$ parameters, $\mathrm{L}$ value in the proposed structure has been shown in Fig. 5. when lumped element resistor value is fixed to $325 \mathrm{ohm}$ and $\mathrm{K}$ value is fixed to $7.6 \mathrm{~mm}$.

After these pre adjustments, L value is simulated between 9.5 and $11.5 \mathrm{~mm}$ in 5 different values and obtained results are given in Fig. 5. As shown in Fig. 5., absorption frequency has been shifted from $4.9 \mathrm{GHz}$ to $6 \mathrm{GHz}$ when $\mathrm{L}$ value is increase from $9.5 \mathrm{~mm}$ to $11 \mathrm{~mm}$. This value is acting an important role on absorption frequency since 
$1 \mathrm{~mm}$ in change result with $1 \mathrm{GHz}$ shift in the resonance frequency which is good tuning option for changing absorption frequency.

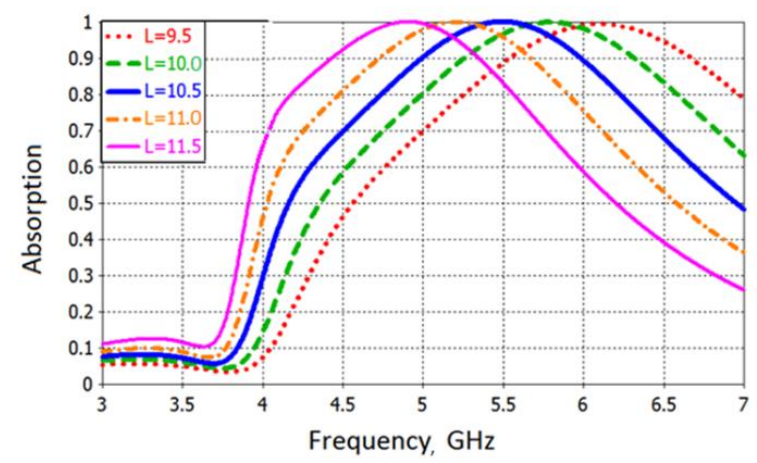

Fig. 5. Parametric result for the $\mathrm{L}$ value, $\mathrm{K}=7.6 \mathrm{~mm}$, $\mathrm{R}=325 \mathrm{ohm}$

Another parameter has been studied by simulation to give more information about design details that is gap value. In this part of study, resistive loads and the dimensions of outer octagonal ring were chosen as $\mathrm{R}=325 \mathrm{ohm}$, $\mathrm{K}=7.6 \mathrm{~mm}$ and $\mathrm{L}=10.5 \mathrm{~mm}$ and gap value is changed between $0.4 \mathrm{~mm}$ to $2 \mathrm{~mm}$ in the same frequency band. As presented in Fig. 6., gap values have been changed from $0.4 \mathrm{~mm}$ to $2 \mathrm{~mm}$ and this change in the gap value results with an absorption frequency shift from $5 \mathrm{GHz}$ to $5.7 \mathrm{GHz}$. After giving information about design details in the proposed structure' usage for WIMAX frequency band operations, In the next section, microwave energy harvesting applications of the proposed structure is discussed.

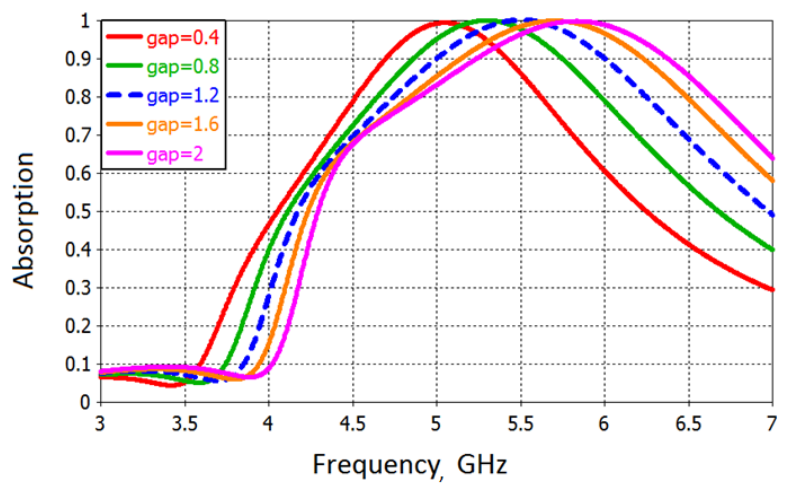

Fig. 6. Parametric results for the gap (mm) with $325 \mathrm{ohm}$ resistive loads

\section{TE, TM AND TEM MODE ANALYSIS}

In this section, the behavior of the proposed octagonal shape metamaterial absorber is investigated in TE, TM and TEM modes. First of all, $S_{11}$ value is investigated when TE polarization is applied to the proposed structure. As shown in Fig. 7., wideband perfect absorption is seen between $5 \mathrm{GHz}$ and $6 \mathrm{GHz}$ which is also WIMAX operation frequency. Absorption value increases to $99.97 \%$ value at $5.5 \mathrm{GHz}$ in TE mode.

In order to show the absorption properties of the proposed structure in other modes as TM and TEM modes, these modes have been simulated by simulation program and obtained results are given in Fig. 8. As shown in Fig. 8., wideband property of the proposed structure under TEM and TM modes cannot be seen between $3 \mathrm{GHz}$ and $7 \mathrm{GHz}$. Single resonance points occur at $6 \mathrm{GHz}$ at $97 \%$ absorption with a bandwidth of $100 \mathrm{MHz}$.

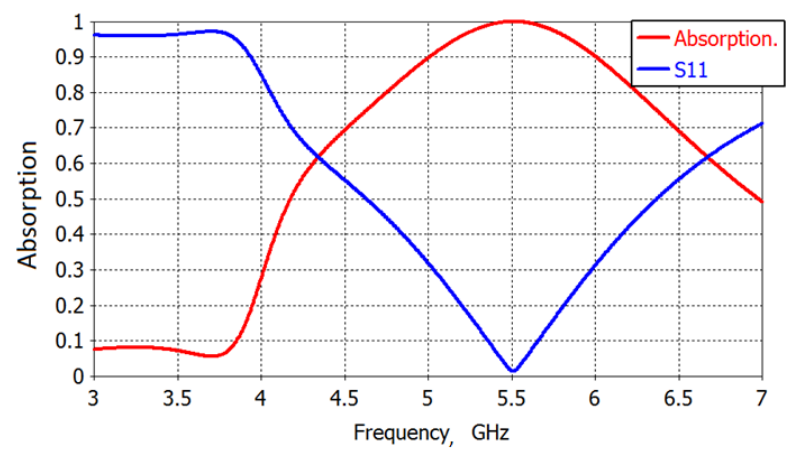

Fig. 7. Absorption and scattering parameter S11 of the proposed structure under TE mode wave

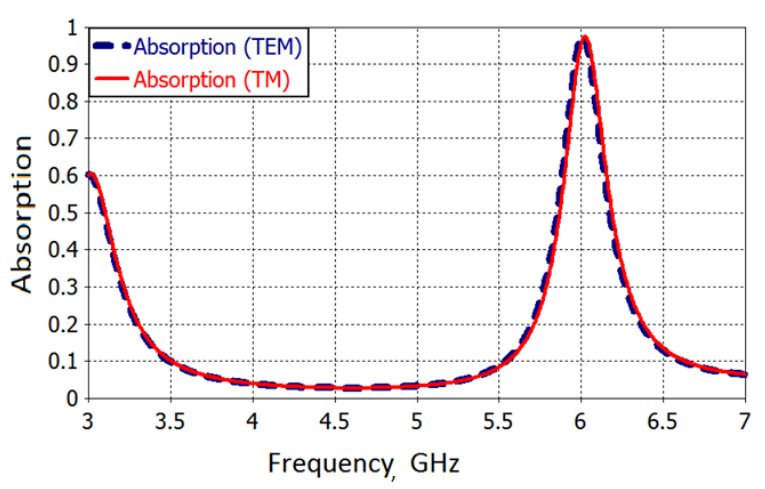

Fig. 8. Absorption of the proposed structure under both TM and TEM modes

Polarization angle independence is another important factor for metamaterial absorber in different polarization angle applications. In order to show the polarization angle effects on absorption, following simulation studies have been realized in TE, TM and TEM mode applications. Obtained results are presented in Fig. 9, Fig. 10 and Fig. 11, respectively.

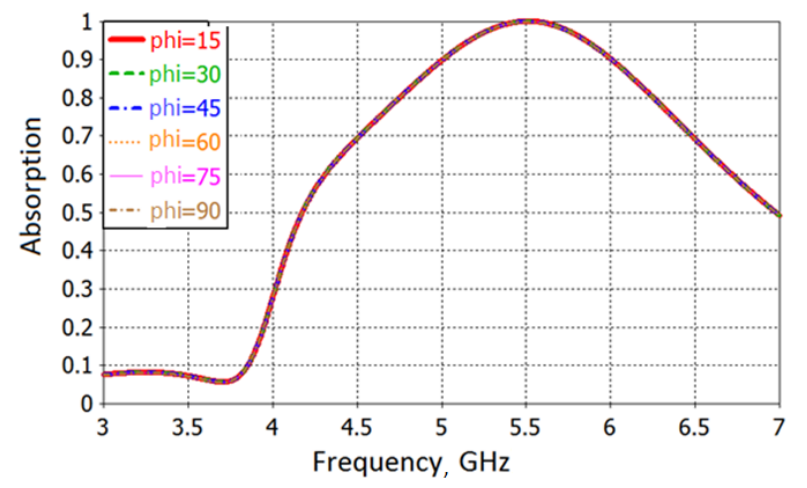

Fig. 9. Absorption under various polarization angle phi for TE mode

When we look at these graphs, we see that phi angle has been adjusted between $0^{\circ}$ and $90^{\circ}$ in 7 different points and 
absorption value is simulated between $3 \mathrm{GHz}$ and 7 GHz.These change in phi angle under the TE, TM and TEM mode applications, do not result with a change in absorption that is a wanted situation for absorber studies.According to Fig. 9, Fig. 10 and Fig. 11, it is clear now that the proposed structure, polarization angle independent under TE, TM and TEM modes.

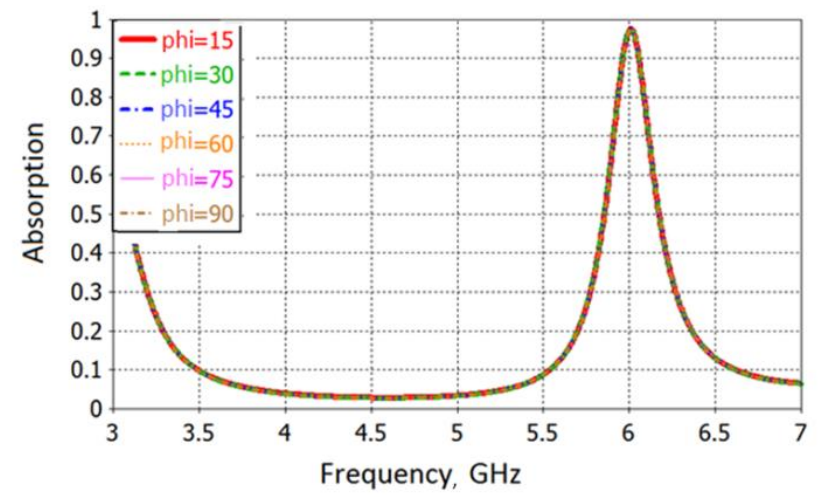

Fig. 10. Absorption under various polarization angle phi for TM mode

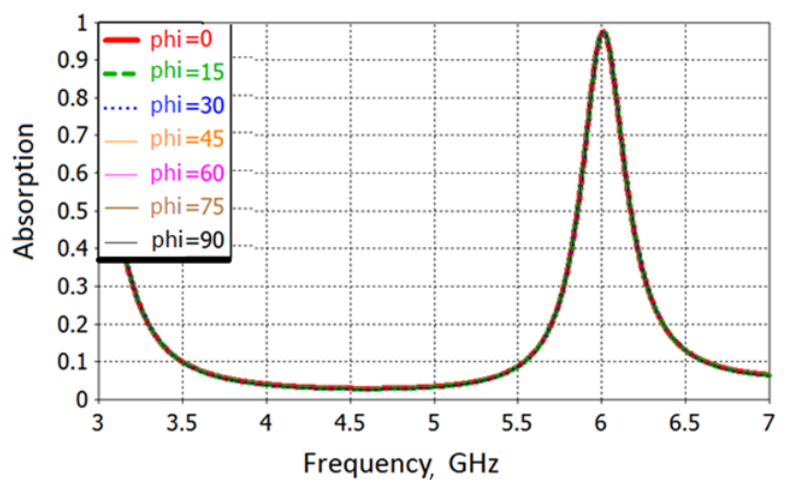

Fig. 11. Absorption under various polarization angle phi for TEM mode

In addition to the polarization angle independency, wide band absorption in TE mode is still protected. These simulation results prove that the proposed structure is a good choice to use in WIMAX frequency portion of microwave spectrum.

\section{ELECTRIC FIELD AND SURFACE CURRENT DISTRIBUTIONS}

In this part of the study, electric field and surface current distributions have been simulated in TE, TM and TEM mode operations. Obtained results are presented in Fig. 12 and Fig. 13. In Fig. 12, electric field distribution is shown under TE mode at $5.5 \mathrm{GHz}$ which has the maximum absorption nearly $99.97 \%$ absorptivity at that frequency. Then, Fig. 12 b and Fig. 12 c, electric field distributions are shown in TM and TEM modes respectively. All graphics are shown in the same scale $(130-190 \mathrm{~dB}-1 \mathrm{uV} / \mathrm{m})$ to see the differences.

When we look at the Fig. 12 a, electric field is concentrated around the splits of the proposed structure which is complying with the direction of electric field electric field is weakening as getting away from centre.

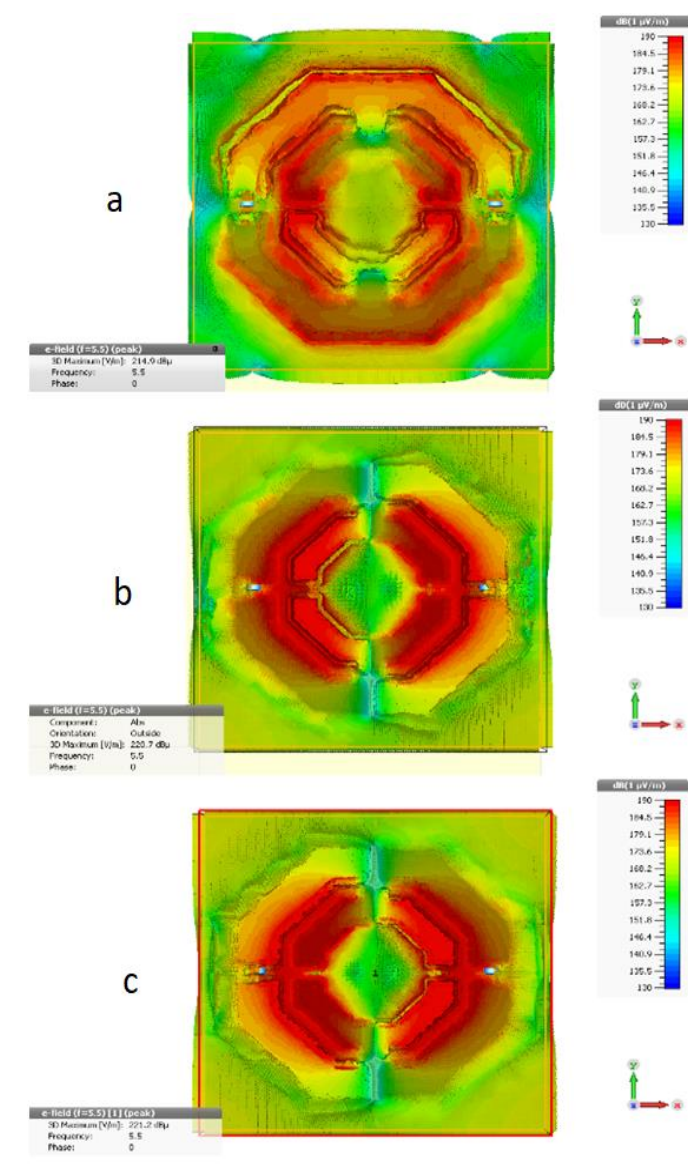

Fig. 12. $a$-electric field distribution for TE mode; $b$ - electric field distribution for TM mode; $\mathrm{c}$-electric field distribution for TEM mode

In TM and TEM modes, electric field distribution is concentrated at left and right side of the structure according to the dimension of the electric and magnetic field.

Surface current distributions of the proposed structure at the resonance frequency of $5.5 \mathrm{GHz}$, is given in Fig. 13 a,b and c, respectively for TE, TM and TEM mode operations.

The electric field component of incident wave excites free electrons as a surface current, surface charge oscillates along the external electric field and this field causes magnetic dipole moment and the dipole moment creates resonance absorption. As it seen from Fig. 13 a, for TE mode, there are parallel and anti-parallel surface currents on the structure. While parallel currents induce electric field, anti-parallel currents result in magnetic field. These responses couple with $\mathrm{E}$ and $\mathrm{H}$ components of the incident electromagnetic wave and produces strong localized electromagnetic field at the resonance frequency. Similar simulation results for TM and TEM mode are also obtained with TE mode operations. According to simulated electric and surface current distributions strong magnetic fields can be seen at the resonance frequencies which is important for energy harvesting applications. In the next sections, energy harvesting performance of the proposed structure and experimental study for reflection coefficient is given.

Resistive elements that have been used as a lumped network element can be used for energy harvesting. For simulation, power on the $325 \mathrm{ohm}$ resistor is chosen as example in TE mode and results are presented in Fig. 14. 


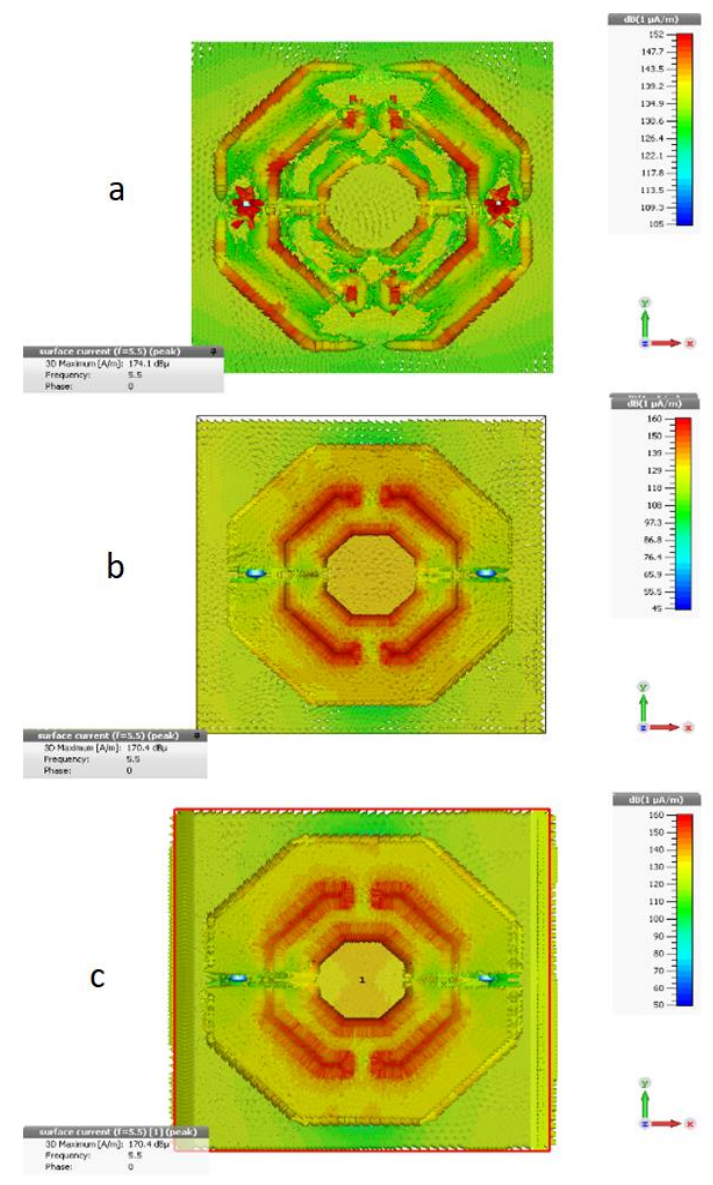

Fig. 13. $a$-surface current distribution for TE mode; $b$ - surface current distribution for TM mode; $\mathrm{c}$-surface current distribution for TEM mode at $5.5 \mathrm{GHz}$

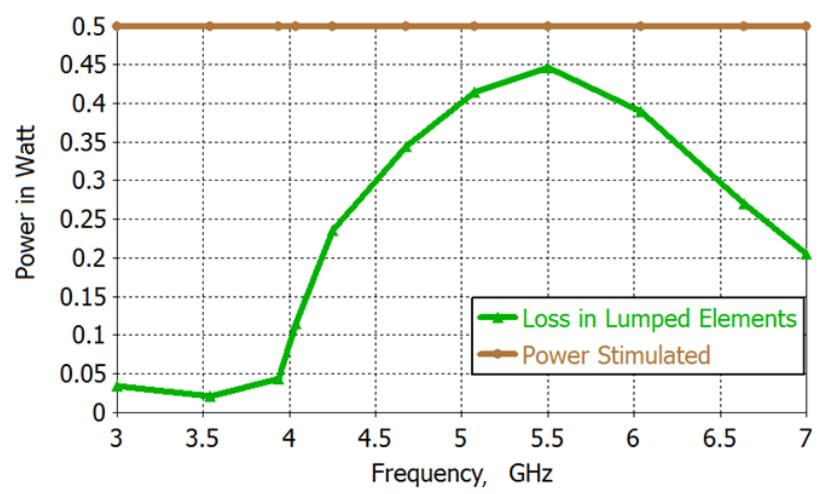

Fig. 14. Simulated power input and energy harvesting efficiency of the proposed structure between 3 and $7 \mathrm{GHz}$ under the TE mode

As it seen from the figure, while $0.5 \mathrm{~W}$ power applied by simulation program between 3 and $7 \mathrm{GHz}, 0.45 \mathrm{~W}$ of the applied signal is harvested again across the lumped element resistor at the frequency of $5.5 \mathrm{GHz}$. Simulated energy harvesting efficiency is found as $90 \%$ at the resonance frequency of $5.5 \mathrm{GHz}$ and it is more than $80 \%$ between 5 and $6 \mathrm{GHz}$. This value decreases to minimum $5 \%$ at $4 \mathrm{GHz}$. Thus, the power on the resistive elements can be used in the harvesting system in microwave frequency region such as, WIMAX bands. In addition to the energy harvesting efficiency between 5 and $6 \mathrm{GHz}$, this values are not affected by polarization change of the applied signal which is also important for energy harvesting applications.

In order to support the energy harvesting application of the proposed structure, we have experimentally tested the proposed structure for reflection coefficient measurements. Results of the experimental study can be used for checking the accuracy of the simulations of the proposed octagonal shape metamaterial absorber. The fabricated structure has 49 unit cells in $7 \times 7$ array shown in the Fig. 15. Promat LPKF E33 CNC controlled PCB production machine is used for the fabrication of the sample which is given in Fig. 15.

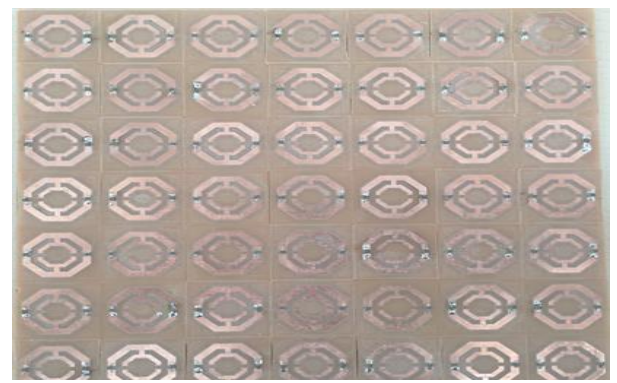

Fig. 15. Fabricated structure

In order to measure reflection coefficient, fabricated sample is placed in front of a horn antenna as shown in Fig. 16 and vector network analyser.

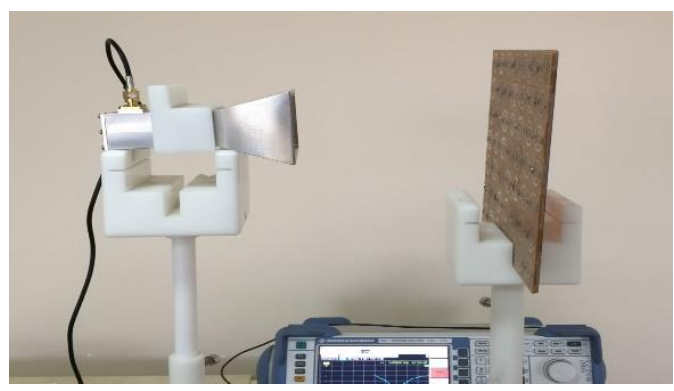

Fig. 16. Experimental setup for TEM mode wave

The free space measurements are obtained by using a horn antenna and a Vector Network Analyzer (VNA). The distance is set to $12.5 \mathrm{~mm}$ which is about $2.5 \lambda$ at $6 \mathrm{GHz}$. Since back side of the structure is covered by copper no transmission occurs on the proposed model. For this reason, measurement of the reflection (S11) is enough for the calculation of the absorptivity of the structure. In the experimental setup, only TEM mode measurements have been completed because of the laboratory capabilities. While measured and simulated reflection coefficient is presented in Fig. 17, absorption values $A(w)$ is given in Fig. 18.

The simulated and measured reflection coefficient resonance frequency have been observed at $6 \mathrm{GHz}$. Simulation and experimental study results are complying with each other and similar results have been obtained.

While simulated reflection coefficient in $\mathrm{dB}$ is $-17 \mathrm{~dB}$ in simulation, the measured reflection coefficient level is defined as $-16 \mathrm{~dB}$ at $6 \mathrm{GHz}$ as expected. In that frequency, $A(w)$ term is calculated as 0.98 which means that $98 \%$ of the incident wave have been absorbed. The simulated and experimental study results show that the proposed structure 
can be used for energy harvesting application in WIMAX frequency band.

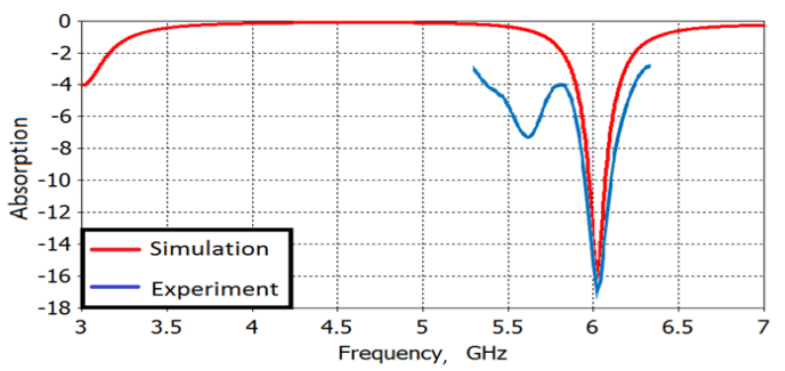

Fig. 17. Reflection (S11 in $\mathrm{dB}$ ) of the simulation and experiment in TEM mode wave

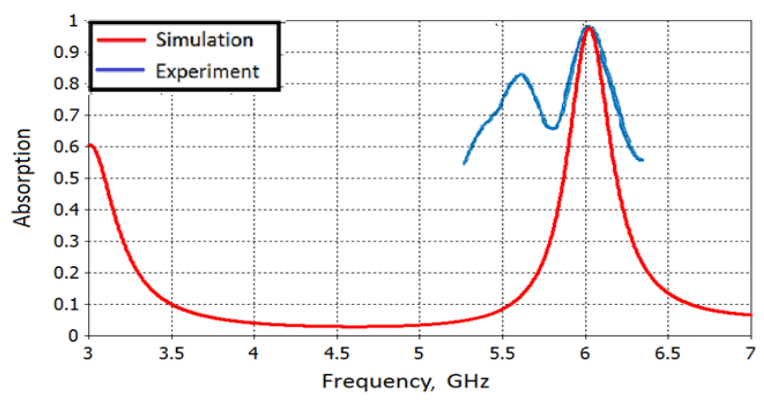

Fig. 18. Absorption of the simulation and the experiment in TEM mode wave

\section{CONCLUSIONS}

Nested octagonal shape metamaterial absorber model is numerically and experimentally designed and investigated in a wide perspective. Design details according to Resistor value, K, L, and gap parameter have been explained by simulation. Simulation results show that it is possible to tune the proposed structure with $1 \mathrm{~mm}$ change in the dimension of L. Also simulation study results show that, maximum energy harvesting occurs when $325 \mathrm{ohm}$ resistor value usage in TE mode. Absorption mechanism is explained by giving electric field and surface current distributions. Energy harvesting efficiency of the proposed structure has been as $80 \%$ between 5 and $6 \mathrm{GHz}$. These results show that the proposed structure can effectively be used as an energy harvester in these bands. Experimental study for supporting the simulated study results have been given for reflection coefficient measurement in TEM mode. The fabricated sample is tested in the facility by using horn antenna and vector network analyser. Obtained results in reflection coefficient measurement show good agreement. According to simulation and experimental study results, the proposed structure can be used as energy harvester in the WIMAX communication band and this has a potential application in the area of absorption and energy harvesting applications. For the future works, this design can be developed for better performance in all application modes as well as sensor applications may be offered.

\section{REFERENCES}

1. Veselago, V.G. The Electrodynamics of Substances with Simultaneously Negative Values of and $\mu$ Soviet Physics Uspekhi 10 (4) 1968: pp. 509.

https://doi.org/10.1070/PU1968v010n04ABEH003699

2. Shelby, R.A., Smith, D.R., Schultz, S. Experimental Verification of a Negative Index of Refraction Science 292 (5514) 2001: pp. $77-79$.

https://doi.org/10.1126/science.1058847

3. Schurig, D., Mock, J.J., Justice, B.J., Cummer, S.A., Pendry, J.B., Starr, A.F., Smith, D.R. Metamaterial Electromagnetic Cloak at Microwave Frequencies Science 314 (5801) 2006: pp. 977 - 980.

https://doi.org/10.1126/science.1133628

4. Pendry, J.B., Schurig, D., Smith, D.R. Controlling Electromagnetic Fields $\quad$ Science $312(5781)$ 2006: pp. $1780-1782$. https://doi.org/10.1126/science.1125907

5. Fang, N., Lee, H., Sun, C., Zhang, X. Sub-diffractionLimited Optical Imaging with a Silver Superlens Science 308 (5721) 2005: pp. 534-537. https://doi.org/10.1126/science.1108759

6. He-Lin, G.L.Y.Y., Chao-Song, L.M.H.G., Yuan, T. A Mircostrip Antenna with Single Square Ring Structured Left-Handed Metamaterial Acta Physica Sinica 1 2012: pp. 021.

https://doi.org/10.7498/aps.61.014102

7. Ziolkowski, R.W., Erentok, A. Metamaterial-Based Efficient Electrically Small Antennas IEEE Transactions on Antennas and Propagation 54 (7) 2006: pp. $2113-2130$. https://doi.org/10.1109/TAP.2006.877179

8. Lim, S., Caloz, C., Itoh, T. Metamaterial-Based Electronically Controlled Transmission-Line Structure as a Novel Leaky-Wave Antenna with Tunable Radiation Angle and Beamwidth IEEE Transactions on Microwave Theory and Techniques 52 (12) 2004: pp. 2678-2690. https://doi.org/10.1109/TMTT.2004.839927

9. Melik, R., Unal, E., Perkgoz, N.K., Puttlitz, C., Demir, H. V.Metamaterial-Based Wireless Strain Sensors Applied Physics Letters 95 (1) 2009: pp. 011106. https://doi.org/10.1063/1.3162336

10. Sabah, C., Dincer, F., Karaaslan, M., Unal, E., Akgol, O.,Demirel, E. Perfect Metamaterial Absorber with Polarization and Incident Angle Independencies Based On Ring and Cross-Wire Resonators for Shielding and a Sensor Application Optics Communications 322 2014: pp. $137-142$. https://doi.org/10.1016/j.optcom.2014.02.036

11. Sabah, C., Roskos, H.G. Broadside-Coupled Triangular Split-Ring-Resonators for Terahertz Sensing The European Physical Journal-Applied Physics 61 (3) 2013: pp. $30402-1: 7$. https://doi.org/10.1051/epjap/2013120053

12. Dincer, F., Karaaslan, M., Colak, S., Tetik, E., Akgol, O., Altıntas, O., Sabah, C. Multi-Band Polarization Independent Cylindrical Metamaterial Absorber and Sensor Application Modern Physics Letters B 30 (08) 2016: pp. 1650095. https://doi.org/10.1142/S0217984916500950

13. Karaaslan, M., Bağmancı, M., Ünal, E., Akgol, O., Sabah, C. Microwave Energy Harvesting Based on Metamaterial Absorbers with Multi-Layered Square Split 
Rings for Wireless Communications Communications 392 2017: pp. 31-38. https://doi.org/10.1016/j.optcom.2017.01.043

14. Dincer, F., Karaaslan, M., Unal, E., Akgol, O. Demirel, E., Sabah, C. Polarization and Angle Independent Perfect Metamaterial Absorber Based On Discontinuous Cross-Wire-Strips Journal of Electromagnetic Waves and Applications 28 (6) 2014: pp. 741-751. https://doi.org/10.1080/09205071.2014.888322

15. Landy, N.I., Sajuyigbe, S., Mock, J.J., Smith, D.R., Padilla, W.J. Perfect Metamaterial Absorber Physical Review Letters 100 (20) 2008: pp. 207402. https://doi.org/10.1103/PhysRevLett.100.207402

16. Sen, G., Banerjee, A., Kumar, M., Islam, S.N., Das, S.A Dual Band Metamaterial Inspired Absorber for WLAN/WiMAX Applications Using a Novel I-Shaped Unit Cell Structure In Microwave Conference (APMC) 2016 AsiaPacific 2016: pp. 1-3. https://doi.org/10.1109/APMC.2016.7931413

17. Sabah, C. Multiband Metamaterials Based On Multiple Concentric Open-Ring Resonators Topology IEEE Journal of Selected Topics in Quantum Electronics 19(1) 2013: pp. $8500808-8500808$. https://doi.org/10.1109/JSTQE.2012.2193875

18. Sabah, C. Novel, Dual Band, Single and Double Negative Metamaterials: Nonconcentric Delta Loop Resonators Progress In Electromagnetics Research B25 2010: pp. $225-239$. https://doi.org/10.2528/PIERB10080302

19. Urbani, F.Experimental Analysis of Novel Single-Sided Left-HandedMetamaterial IEEE Antennas and Wireless Propagation Letters 9 2010: pp. 720-723. https://doi.org/10.1109/LAWP.2010.2058837
20. Fernandes, H.C.C. Metamaterial Antennas and Finlines Using Full Wave Analysis In WSEAS International Conference. Proceedings. Mathematics and Computers in Science and Engineering 2008: pp. 69-73.

21. Huang, L., Chen, $\mathbf{H}$. Multi-Band and Polarization Insensitive Metamaterial Absorber Progress In Electromagnetics Research 113 2011: pp. 103-110. https://doi.org/10.1063/1.4878697

22. Bilotti, F., Nucci, L., Vegni, L. An SRR Based Microwave Absorber Microwave and Optical Technology Letters 48 (11) 2006: pp. 2171-2175. https://doi.org/10.1002/mop.21891

23. Zhu, B., Wang, Z., Huang, C., Feng, Y., Zhao, J., Jiang, T. Polarization Insensitive Metamaterial Absorber with WideIncident Angle Progress In Electromagnetics Research 101 2010: pp. 231-239. https://doi.org/10.2528/PIER10011110

24. Dincer, F., Karaaslan, M., Unal, E., Delihacioglu, K., Sabah, C. Design of Polarization and Incident Angle Insensitive Dual-Band Metamaterial Absorber Based On Isotropic Resonators Progress In Electromagnetics Research 144 2014: pp. 123-132. https://doi.org/10.2528/PIER13111403

25. Cheng, Y., Yang, H. Design, Simulation, and Measurement of Metamaterial Absorber Journal of Applied Physics 108 (3) 2010: pp. 034906. https://doi.org/10.1063/1.3311964

26. Xie, Y., Fan, X., Chen, Y., Wilson, J.D., Simons, R.N., Xiao, J.Q. A Subwavelength Resolution Microwave/6.3 Ghz Camera Based on a Metamaterial Absorber Scientific Reports 7 2017: pp. 1-8. https://doi.org/doi:10.1038/srep40490 